\title{
An Unusual Cause of Syncope With T-Wave Abnormalities
}

Michael J. Bono, MD; Francis L. Counselman, MD

\section{A 34-year-old man presented for evaluation of syncope. He had a headache and had been vomiting over the past week.}

\begin{abstract}
Case
A 34-year-old man presented to our ED via emergency medical services (EMS) following a syncopal episode. The patient stated that as he was getting ready for work earlier that morning, he experienced sudden lightheadedness and passed out, whereupon his wife immediately called EMS. The patient denied any previous history of syncope, but said he had been experiencing frequent episodes of nausea and vomiting over the past week. He also complained of a mild occipital headache that acetaminophen had failed to relieve.

The patient had been seen at a different ED 3 days earlier for nausea and vomit-
\end{abstract}

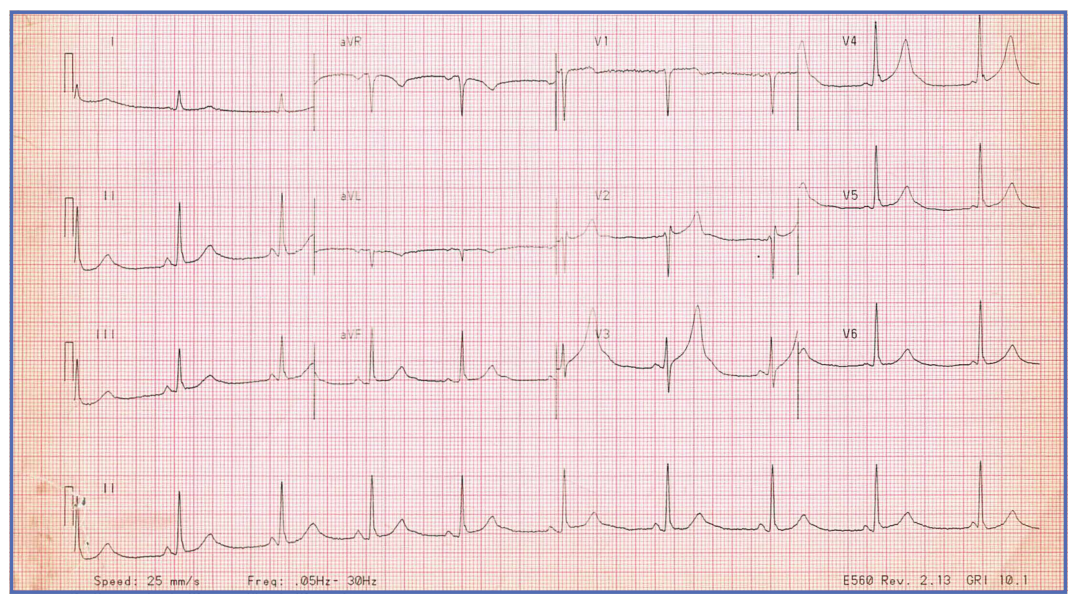

Figure. Electrocardiogram taken of patient demonstrating sinus bradycardia, an incomplete right bundle branch block, and tall, splayed upright T waves in the precordial leads. ing. After evaluating the patient, the emergency physician (EP) at this facility felt the most likely cause of the patient's gastrointestinal issues was related to hydralazine, his antihypertensive medication, and advised the patient to discontinue its use.

During evaluation at our ED, the patient denied fever, chills, neck stiffness, numbness, weakness, tingling of the extremities, or difficulty walking. He also denied chest pain, shortness of breath, or urinary symptoms. The patient's medical history was significant only for hypertension; he had not taken any antihypertensive or other medications for the past 3 days, as previously instructed by the EP at the other ED. The patient denied alcohol or drug abuse.

On physical examination, the patient's vital signs were: temperature, $98.6^{\circ} \mathrm{F}$; heart rate, 58 beats/minute; blood pressure, 130/90 mm Hg; and respiratory rate, 16 breaths/minute. Oxygen saturation was $100 \%$ on room air. Examination of the head was normal and without evidence of trauma. Both pupils measured $4 \mathrm{~mm}$ and were equally round and reactive to light; the patient's extraocular movements were intact. The remainder of the head, eyes, ears, nose, and throat examination was normal. The neck was supple, without masses or meningeal signs. The cardiopulmonary and abdominal examinations were all normal. On neurological examination, the patient was awake, alert, and oriented

Dr Bono is a professor and vice chairman, department of emergency medicine, Eastern Virginia Medical School, Norfolk. Dr Counselman is the distinguished professor and chairman of the department of emergency medicine at Eastern Virginia Medical School. He is also the associate editor in chief of EMERGENCY MEDICINE. Both authors are physicians at Emergency Physicians of Tidewater, Norfolk. 
to person, place, and time. Cranial nerves II through XII were intact, and the patient had 5/5 motor strength in all four extremities and a normal gait.

Because we were concerned about the patient's unexplained syncopal episode, we ordered laboratory tests, including a complete blood count (CBC), evaluation of electrolytes and glucose levels, and kidney function. In addition, we also ordered an electrocardiogram (ECG) and a noncontrast computed tomography (CT) scan of the head. All laboratory test results were within normal range. The ECG, however, demonstrated sinus bradycardia (approximately 58 beats/minute), a normal PR and QRS interval, a normal axis, and an incomplete right bundle branch block with tall, large, splayed upright $\mathrm{T}$ waves in the precordial leads (Figure). Based on the abnormal ECG results, we ordered serum cardiac marker studies, the values of which were all within normal range. The noncontrast CT scan of the head revealed a low-density posterior fossa mass compressing the fourth ventricle with secondary hydrocephalus.

The patient was placed with his head in an upright position and given $1 \mathrm{~g} / \mathrm{kg}$ mannitol and $10 \mathrm{mg}$ dexamethasone intravenously (IV). Neurosurgery services were consulted, and the patient underwent surgery the following morning. Surgery confirmed the presence of a hemangioblastoma. The hemangioblastoma was successfully excised, and the patient had an uneventful recovery. Interestingly, the significant $\mathrm{T}$ wave changes in the precordial leads were no longer present postoperatively.

\section{Discussion}

Syncope and near-syncope are common reasons for ED visits. Syncope is a syndrome characterized by a transient, selflimited episode of loss of consciousness occurring as a result of a brief interruption of the oxygen supply to the brain. ${ }^{1}$ This interruption is almost always due to a transient cessation of blood flow. ${ }^{1}$ In true syncope (as opposed to seizures or hypo-
Table. Diagnostic Classification of the Causes of Syncope ${ }^{a}$

\author{
Reflex (Neurally-Mediated) Syncope \\ Vasovagal \\ Triggered by emotional stress \\ Triggered by orthostatic stress \\ Situational \\ Cough, sneeze \\ Gastrointestinal stimulation \\ Micturition \\ Carotid sinus syncope
}

\section{Orthostatic Hypotension Syncope}

Volume depletion: Inadequate fluid intake, vomiting, diarrhea, etc

Drug-induced: Alcohol, vasodilators, diuretics, beta-adrenergic blockers

Primary autonomic failure (pure autonomic failure, multiple system atrophy, Parkinson disease with autonomic failure, Lewy body dementia)

Secondary autonomic failure (diabetes mellitus, amyloidosis, spinal cord injuries)

\section{Cardiac Syncope (Cardiovascular) Arrhythmia as Primary Cause \\ Bradycardia \\ Sinus node dysfunction, atrioventricular conduction system disease Implanted device malfunction \\ Tachycardia \\ Supraventricular \\ Ventricular}

\section{Structural Disease}

Cardiac (valvular disease, infarction/ischemia, hypertrophic cardiomyopathy, cardiac tumor, pericardial disease/tamponade, prosthetic valve dysfunction)

Other (pulmonary embolism/hypertension, aortic dissection)

aModified from the European Society of Cardiology Syncope Practice Guidelines.

Reprinted from Puppala VK, Dickinson O, Benditt DG. Syncope: classification and risk stratification. J Cardiol. 2014;63(3):171-177, with permission from Elsevier. ${ }^{1}$

glycemia), the episode is characterized by a rapid onset of loss of consciousnesswith or without warning symptoms. ${ }^{1}$ It is important to determine the cause of syncope, because $7 \%$ to $23 \%$ of such patients will suffer serious outcomes within 7 to 30 days of their ED visit-either within a hospital setting or at home. ${ }^{2}$

\section{Etiology}

There are many causes of syncope. In most cases, the etiology falls under one of three broad categories: neurally mediated (or 
reflex mediated), orthostatic hypotensivemediated, or cardiovascular (CV)-mediated. Less common causes of syncope include cerebrovascular injury. ${ }^{1}$ The Table outlines both common and uncommon causes of syncope.

On presentation, our patient had several possible causes for his syncopal episode: an abnormal ECG (CV); multiple episodes of emesis (volume depletion); and headache (cerebrovascular). The EP worked up all three of these signs and symptoms simultaneously as is the appropriate protocol when evaluating an ED patient presenting with undifferentiated syncope.

\section{Signs and Symptoms}

Patients with undiagnosed brain tumors normally present with headache, seizures, nausea, vomiting, focal neurological deficits, or an altered mental status. ${ }^{3}$ Syncope is a very rare manifestation of a brain tumor $^{3}$; however, our patient did complain of headache, nausea, and vomiting.

In addition to the unusual cause of the syncope, the abnormally large upright $\mathrm{T}$ waves make this case even more notable. T-wave changes are the most common ECG abnormality, seen in about $50 \%$ of abnormal tracings reviewed in a hospital population and in $2.4 \%$ of all ECGs. ${ }^{4}$

In general, T-wave changes are a result of local changes in the duration of repolarization. T-wave inversion is the most common T-wave abnormality and is typically observed in the setting of ischemia, postingestion of food, following an episode of tachycardia or anxiety, and autonomic dysfunction. ${ }^{5}$ However, in patients who have a cerebral etiology (usually hemorrhage), the T-wave changes may be either upright (as in our case) or inverted. ${ }^{5}$ Historically, subarachnoid hemorrhage (SAH) has been associated with ST-segment elevation and T-wave inversion. Hypothalamic stimulation and autonomic dysfunction have been linked to abnormal T-waves, but this has not been conclusively proven to be the cause of the abnormality. ${ }^{6}$ For all of the aforementioned reasons, the specificity for a given cause of T-wave changes is exceedingly low. ${ }^{5}$

Hyperacute T-wave amplitude, with prominent symmetrical $\mathrm{T}$ waves in at least two continuous leads, may be the earliest sign of acute transmural myocardial infarction (MI). ${ }^{7}$ It usually persists for only a brief time before other ECG findings of acute MI are observed. Other common causes of hyperacute $\mathrm{T}$ waves include hyperkalemia (usually narrow-based, and peaked), early repolarization, left ventricular hypertrophy, and acute myocarditis. ${ }^{8}$ Less common causes of prominent $\mathrm{T}$ waves include preexcitation syndromes, pericarditis, and scorpion stings. ${ }^{9,10}$

\section{Summary}

It is unclear why our patient, who had a hemangioblastoma, demonstrated hyperacute T-wave abnormality on ECG. The abnormal upright $\mathrm{T}$ waves may have occurred secondary to the same theories regarding $\mathrm{SAH}$, hypothalamic stimulation, or autonomic dysfunction. Regardless of the underlying etiology, this case serves as a reminder to the EP that not all T-wave changes on ECG are cardiac in origin.

\section{References}

1. Puppala VK, Dickinson O, Benditt DG. Syncope: classification and risk stratification. J Cardiol. 2014;63(3):171-177.

2. Thiruganasambandamoorthy V, Stiell IG, Sivilotti $\mathrm{ML}$, et al. Risk stratification of adult emergency department syncope patients to predict short-term serious outcomes after discharge (RiSEDS) study. BMC Emerg Med. 2014;14(1):8.

3. van der Sluijs BM, Renier WO, Kappelle AC. Brain tumor as a rare cause of cardiac syncope. J Neurooncol. 2004;67(1-2):241-244.

4. Friedberg CK, Zagar A. Nonspecific ST and T-wave changes. Circulation. 1961;23:665-661.

5. Fisch C. T wave abnormalities due to extracardiac "functional" causes. ACC Curr J Rev. 1997;6(2):101-104.

6. Chatterjee S. ECG changes in subarachnoid hemorrhage: a synopsis. Neth Heart J. 2011;19(1):31-47.

7. Vojáčeka, J, Janskýb P, Janotac T. Third universal definition of myocardial infarction. Cor Vasa. 2013;55:e228-e235.

8. Levis JT. ECG diagnosis: hyperacute T waves. Perm J. 2015;19(3):79.

9. Somers MP, Brady WJ, Perron AD, Mattu A. The prominent T wave: electrocardiographic differential diagnosis. Am J Emerg Med. 2002;20(3):243-251.

10. Kumar MR, Bharath RV, Subrahmanyam BV, Rammohan P, Agrawal A. Scorpion envenomation and its management in adults. Sahel Med J. 2013;16(2):60-63. 